IMA Journal of Numerical Analysis (2005) Page 1 of 16

doi: 10.1093/imanum/dri017

\title{
Variational-Splitting Time Integration of the Multi-Configuration Time-Dependent Hartree-Fock Equations in Electron Dynamics
}

\author{
OTHMAR KOCH \\ Vienna University of Technology, Austria \\ CHRISTIAN LUBICH \\ University of Tübingen, Germany
}

\begin{abstract}
We discuss the numerical approximation of the solution to the multi-configuration time-dependent Hartree-Fock (MCTDHF) equations in quantum dynamics. The MCTDHF method approximates the high-dimensional wave function of the time-dependent electronic Schrödinger equation by an antisymmetric linear combination of products of functions depending only on three-dimensional spatial coordinates. The equations of motion, obtained via the Dirac-Frenkel time-dependent variational principle, consist of a coupled system of three-dimensional nonlinear partial differential equations and ordinary differential equations. We investigate the convergence properties of a time integrator based on a splitting of the Hamiltonian directly in the variational principle. First-order convergence in the $H^{1}$ Sobolev norm and second-order convergence in the $L^{2}$ norm are established under a solution regularity of $H^{2}$. As a prerequisite, we show that the MCTDHF equations have a solution in this Sobolev space if the initial data has such regularity.
\end{abstract}

Keywords: MCTDHF method, electronic Schrödinger equation, splitting methods, regularity.

\section{Introduction}

This paper deals with an approach to the approximate solution of the time-dependent Schrödinger equation for a system of unbound fermions interacting by Coulomb forces,

$$
\mathrm{i} \frac{\partial \psi}{\partial t}=\mathbf{H} \psi, \quad \psi(0)=\psi_{0},
$$

where the wave function $\psi=\psi\left(x^{(1)}, \ldots, x^{(d)}, t\right)$ depends on the spatial coordinates $x^{(k)} \in \mathbb{R}^{3}$ of $d$ particles, and on time $t$. The many-body Hamiltonian is given by

$$
\mathbf{H}=\mathbf{T}+\mathbf{V},
$$

where the kinetic energy operator $\mathbf{T}$ is the sum of the kinetic energy operators of the particles,

$$
\mathbf{T}=\sum_{k=1}^{d} T^{(k)} \quad \text { with } T^{(k)}=-\frac{1}{2} \Delta_{x^{(k)}}
$$

with the Laplacian $\Delta_{x^{(k)}}$ with respect to the coordinates of the $k$ th particle, and where the potential $\mathbf{V}$ is given by pairwise Coulomb interaction of the particles,

$$
\mathbf{V}=\sum_{l<k} \frac{1}{\left|x^{(k)}-x^{(l)}\right|},
$$


with the Euclidean norm $|\cdot|$ in $\mathbb{R}^{3}$.

The applications that motivate this research are given by the study of ultrafast laser pulses in photonics $[5,14,29,30]$. There, the single-particle parts of the Hamiltonian additionally depend on a time-dependent drift term modeling the laser determined by a real-valued function $A(t)$, and moreover an attractive potential to a nucleus of charge $Z$ at the origin is incorporated,

$$
T^{(k)}=\frac{1}{2}\left(-\mathrm{i} \nabla_{x^{(k)}}-A(t)\right)^{2}-\frac{Z}{\left|x^{(k)}\right|} .
$$

Here and in the following, the subscript of the differential operator $\nabla_{x^{(k)}}$ (and likewise for $\Delta_{x^{(k)}}$ ) refers to differentiation with respect to the space variables associated with the $k$ th particle. Extensions of our analysis to models (1.3) are briefly discussed at the end of Sections 3 and 4.

The method chosen to make the linear electronic Schrödinger equation (1.1) tractable for numerical computation, is the multi-configuration time-dependent Hartree-Fock method, MCTDHF [5, 13, 14, 25, 29, 30], which is closely related to the MCTDH method in quantum molecular dynamics [2, 3, 4, 23, 24$]$.

In the MCTDHF approach, the wave function is approximated by a linear combination of antisymmetrized products (Slater determinants) of functions each depending on the coordinates of only a single particle (henceforth referred to as orbitals). The antisymmetry is required to concur with the Pauli exclusion principle [19]. The Dirac-Frenkel time-dependent variational principle [8, 9] yields equations of motion for the orbitals and the coefficients in the linear combination of the products. The MCTDHF method thus replaces the high-dimensional linear Schrödinger equation by a system of low-dimensional nonlinear partial differential equations and ordinary differential equations and in this way makes the problem computationally tractable.

In Section 3, we study the nonlinear equations of motion of the MCTDHF approach. For initial data in the Sobolev space $H^{2}$ of functions that are square integrable together with all their first and secondorder weak partial derivatives, we show that a unique solution to the MCTDHF equations exists in $H^{2}$, even globally in time unless a density matrix appearing in the method formulation becomes singular.

In Section 4, we study the approximation of the MCTDHF equations by semi-discretization in time employing an operator splitting introduced in [20], which splits the Hamiltonian directly on the level of the time-dependent variational principle. It is shown that for a symmetric, second-order splitting, firstorder convergence holds in $H^{1}$ when the solution is in $H^{2}$, and the method is second-order convergent in $L^{2}$ for the same solution regularity.

\section{The MCTDHF method}

In the MCTDHF method, the multi-particle wave function $\psi$ is approximated by an antisymmetric linear combination of Hartree products, that is,

$$
\psi\left(x^{(1)}, \ldots, x^{(d)}, t\right) \approx u\left(x^{(1)}, \ldots, x^{(d)}, t\right)=\sum_{\left(j_{1}, \ldots, j_{d}\right)} a_{j_{1}, \ldots, j_{d}}(t) \phi_{j_{1}}\left(x^{(1)}, t\right) \cdots \phi_{j_{d}}\left(x^{(d)}, t\right) .
$$

Here, the multi-indices $J=\left(j_{1}, \ldots, j_{d}\right)$ formally vary for $j_{k}=1, \ldots, N, k=1, \ldots, d$, the $a_{J}(t)$ are complex coefficients depending only on $t$, and the $N$ orbitals $\phi_{j}(x, t)$ depend on the three-dimensional spatial variable $x \in \mathbb{R}^{3}$ and on time $t$. In view of the Pauli principle, antisymmetry is imposed on the coefficients $a_{J}$ : for any permutation $\sigma$ of $\{1, \ldots, n\}$, we have with $\sigma(J)=\left(j_{\sigma(1)}, \ldots, j_{\sigma(n)}\right)$ that

$$
a_{\sigma(J)}(t)=\operatorname{sign}(\sigma) a_{J}(t) \quad \text { for all } t \text {. }
$$


The approximation can thus alternatively be written as

$$
u\left(x^{(1)}, \ldots, x^{(d)}, t\right)=\sum_{1 \leqslant j_{1}<\cdots<j_{d} \leqslant N} a_{j_{1}, \ldots, j_{d}}(t) \sqrt{d !} \phi_{j_{1}} \wedge \cdots \wedge \phi_{j_{d}}\left(x^{(1)}, \ldots, x^{(d)}, t\right)
$$

with the antisymmetrized products (Slater determinants)

$$
\phi_{j_{1}} \wedge \cdots \wedge \phi_{j_{d}}\left(x^{(1)}, \ldots, x^{(d)}, t\right)=\frac{1}{\sqrt{d !}} \operatorname{det}\left(\phi_{j_{k}}\left(x^{(l)}, t\right)\right)_{k, l=1, \ldots, d} .
$$

Thus, in fact only $\left(\begin{array}{l}N \\ d\end{array}\right)$ coefficients $a_{J}$ have to be determined in the actual computations. The MCTDHF method is a model reduction analogous to the low rank approximation of time-dependent matrices, where a large system matrix is replaced by a linear combination of rank-1 matrices. The particular choice $N=d$, that is, taking only a single Slater determinant for the approximation, is known as the time-dependent Hartree-Fock method (TDHF). It dates back to Dirac [8] and has since long found widespread use in physics.

The Dirac-Frenkel time-dependent variational approximation principle [8, 9] (see also [22] for a detailed discussion) is used to derive differential equations for the coefficients $a_{J}$ and the orbitals $\phi_{j}$ in (2.1). For $u(t)$ in the manifold $\mathscr{M}$ of ansatz functions (2.1) of full rank, we require that the time derivative $\partial u / \partial t$ be chosen such that the residual of the Schrödinger equation is orthogonal to the tangent space $\mathscr{T}_{u} \mathscr{M}$ of $\mathscr{M}$ at $u$ :

$$
\left\langle\delta u \mid \mathrm{i} \frac{\partial u}{\partial t}-\mathbf{H} u\right\rangle=0 \quad \text { for all } \delta u \in \mathscr{T}_{u} \mathscr{M} .
$$

Using the Dirac-Frenkel principle and imposing orthogonality and gauge conditions on the orbitals,

$$
\begin{aligned}
& \left\langle\phi_{j} \mid \phi_{k}\right\rangle=\delta_{j, k}, \quad t \geqslant 0, \\
& \left\langle\phi_{j} \mid \mathrm{i} \frac{\partial \phi_{l}}{\partial t}\right\rangle=\left\langle\phi_{j}|T| \phi_{l}\right\rangle, \quad t \geqslant 0,
\end{aligned}
$$

with $T=-\frac{1}{2} \Delta$, yields a system of coupled ordinary and partial differential equations for the coefficients $a=\left(a_{J}\right)_{J}$ and orbitals $\phi=\left(\phi_{j}\right)_{j}$, rigorously derived in $[2,23]$ under the implicit assumption that a sufficiently regular solution exists:

$$
\begin{aligned}
\mathrm{i} \frac{d a_{J}}{d t} & =\sum_{L}\left\langle\Phi_{J}|\mathbf{V}| \Phi_{L}\right\rangle a_{L} \quad \forall J \\
\mathrm{i} \frac{\partial \phi_{j}}{\partial t} & =T \phi_{j}+(I-P) \sum_{l=1}^{N} \sum_{m=1}^{N} \rho_{j, m}^{-1}\left\langle\psi_{m}|\mathbf{V}| \psi_{l}\right\rangle \phi_{l}, \quad j=1, \ldots, N
\end{aligned}
$$

Here we have the Hartree products $\Phi_{J}:=\bigotimes_{k=1}^{n} \phi_{j_{k}}$ and the single-hole functions

$$
\psi_{j}:=\left\langle\phi_{j} \mid u\right\rangle, \quad j=1, \ldots, N
$$

with the integration in the $L^{2}$ inner product over the first variable $x^{(1)}$, so that by (2.6),

$$
\psi_{j}\left(x^{(2)}, \ldots, x^{(d)}, t\right)=\sum_{\left(j_{2}, \ldots, j_{d}\right)} a_{j, j_{2}, \ldots, j_{d}}(t) \phi_{j_{2}}\left(x^{(2)}, t\right) \ldots \phi_{j_{d}}\left(x^{(d)}, t\right) .
$$


The inner products $\left\langle\psi_{m}|\mathbf{V}| \psi_{l}\right\rangle$ are over all remaining variables. Since $\mathbf{V}$ is a sum of two-particle interactions, these high-dimensional integrals as well as those in (2.8) actually reduce to six- and threedimensional integrals.

The density matrix $\rho(t)$ with entries

$$
\rho_{j, l}:=\left\langle\psi_{j} \mid \psi_{l}\right\rangle=\sum_{\left(j_{2}, \ldots, j_{d}\right)} \bar{a}_{j, j_{2}, \ldots, j_{d}} a_{l, j_{2}, \ldots, j_{d}}
$$

is assumed to be nonsingular, and $\rho_{j, m}^{-1}$ in (2.9) are the entries of $\rho^{-1}$. The choice of the initial condition such that $\rho$ is nonsingular ensures that this holds at least for small $t$. In [1] a sufficient criterion for global invertibility of $\rho$ is given.

Finally, $P$ is the orthogonal projector onto the space spanned by $\phi_{1}, \ldots, \phi_{N}$,

$$
P \chi=\sum_{j=1}^{N} \phi_{j}\left\langle\phi_{j} \mid \chi\right\rangle \text {. }
$$

The problem formulation based on the gauge condition (2.7) offers the advantage that the single-particle operator $T=-\frac{1}{2} \Delta$ appears outside the projection in (2.9).

\section{Regularity of MCTDHF orbitals}

As a prerequisite for the convergence analysis of numerical approximations, we establish the existence of a unique, regular solution to the MCTDHF equations (2.8)-(2.9).

THEOREM 3.1 Consider the system (2.8)-(2.9) together with initial conditions such that the orthonormality constraints (2.6) are satisfied and the density matrix $\rho(0)$ defined in (2.11) is nonsingular. If the initial data for $\phi_{j}$ is in the Sobolev space $H^{2}$, then there exists a unique strong solution of the MCTDHF equations (2.8)-(2.9) satisfying

$$
a_{J} \in C^{2}\left(\left[0, t^{*}\right), \mathbb{C}\right), \quad \phi_{j} \in C^{1}\left(\left[0, t^{*}\right), L^{2}\right) \cap C\left(\left[0, t^{*}\right), H^{2}\right),
$$

where either $t^{*}=\infty$ or the density matrix $\rho(t)$ becomes singular for $t \uparrow t^{*}$. Moreover, for $u$ defined by $a_{J}, \phi_{j}$ via (2.1), we have $u(t) \in H^{2}$ for $t \in\left[0, t^{*}\right)$, and $u$ solves the Dirac-Frenkel variational equation (2.5).

For the single-configuration time-dependent Hartree-Fock method (TDHF), a corresponding result was given by Chadam and Glassey [7]. In parallel to our work, an existence result for the MCTDHF equations was recently given in [1], including also a criterion for the global invertibility of the density matrix $\rho$. Since the estimates needed for our argument are the same as those required in the convergence analysis of the variational splitting given in Section 4, we nevertheless include our analysis in this section.

To prepare for the proof of Theorem 3.1, we note that the right-hand side of (2.8)-(2.9) contains terms of the forms ${ }^{1}$

$$
\begin{aligned}
& \left\langle\phi_{1}(x)\left|\frac{1}{|x-y|}\right| \phi_{2}(x)\right\rangle_{L^{2}(x)} \tilde{\phi}_{2}(y)=: \mathscr{F}(y), \\
& \left\langle\phi_{1}(x) \tilde{\phi}_{1}(y)\left|\frac{1}{|x-y|}\right| \phi_{2}(x) \tilde{\phi}_{2}(y)\right\rangle_{L^{2}(x, y)}=: \mathscr{J},
\end{aligned}
$$

\footnotetext{
${ }^{1}$ The subscripts on inner products and norms refer to the function spaces involved and the variables these are associated with. They will be omitted if there is no danger of ambiguity.
} 
which need to be estimated. These are associated with the particle-particle interactions $\mathbf{V}$. Note that (3.2) appears only in the equations (2.8) since in (2.9) these contributions are annihilated by the projection $I-P$, and that the terms actually take the form of antisymmetric linear combinations

$$
\begin{aligned}
& \left\langle\phi_{1}(x)\left|\frac{1}{|x-y|}\right| \phi_{2}(x) \tilde{\phi}_{2}(y)-\tilde{\phi}_{2}(x) \phi_{2}(y)\right\rangle_{L^{2}(x)}, \\
& \left\langle\phi_{1}(x) \tilde{\phi}_{1}(y)\left|\frac{1}{|x-y|}\right| \phi_{2}(x) \tilde{\phi}_{2}(y)-\tilde{\phi}_{2}(x) \phi_{2}(y)\right\rangle_{L^{2}(x, y)}
\end{aligned}
$$

The following lemma collects the necessary estimates.

LEMma 3.1 Let $\mathscr{F}$ and $\mathscr{J}$ be defined by (3.1)-(3.2). Then, there are the following bounds with a constant $C$ independent of $\phi_{1}, \phi_{2}, \tilde{\phi}_{1}, \tilde{\phi}_{2}$.

$$
\begin{aligned}
\|\mathscr{F}\|_{L^{2}} & \leqslant C\left\|\phi_{1}\right\|_{L^{2}}\left\|\phi_{2}\right\|_{H^{1}}\left\|\tilde{\phi}_{2}\right\|_{L^{2}} \\
\|\nabla \mathscr{F}\|_{L^{2}} & \leqslant C\left(\left\|\phi_{1}\right\|_{H^{1}}\left\|\phi_{2}\right\|_{H^{1}}\left\|\tilde{\phi}_{2}\right\|_{L^{2}}+\left\|\phi_{1}\right\|_{L^{2}}\left\|\phi_{2}\right\|_{H^{1}}\left\|\tilde{\phi}_{2}\right\|_{H^{1}}\right) \\
\|\Delta \mathscr{F}\|_{L^{2}} & \leqslant C\left\|\phi_{1}\right\|_{H^{1}}\left\|\phi_{2}\right\|_{H^{1}}\left\|\tilde{\phi}_{2}\right\|_{H^{2}} \\
|\mathscr{J}| & \leqslant C\left\|\phi_{1}\right\|_{L^{2}}\left\|\phi_{2}\right\|_{H^{1}}\left\|\tilde{\phi}_{1}\right\|_{L^{2}}\left\|\tilde{\phi}_{2}\right\|_{L^{2}} .
\end{aligned}
$$

Moreover, the dependence of $\mathscr{F}$ and $\mathscr{J}$ on $\phi_{1}, \phi_{2}, \tilde{\phi}_{1}, \tilde{\phi}_{2}$ is locally Lipschitz continuous from $H^{2}$ to $H^{2}$ and from $H^{1}$ to $\mathbb{C}$, respectively.

Proof. Much of the proof rests on the Hardy inequality [12, p.350]

$$
\int_{\mathbb{R}^{3}} \frac{|u(y)|^{2}}{|x-y|^{2}} d y \leqslant 4 \int_{\mathbb{R}^{3}}|\nabla u(y)|^{2} d y, \quad x \in \mathbb{R}^{3} .
$$

The Cauchy-Schwarz inequality and the Hardy inequality yield

$$
\left\|\left\langle\phi_{1}(x)\left|\frac{1}{|x-y|}\right| \phi_{2}(x)\right\rangle_{L^{2}(x)}\right\|_{L^{\infty}(y)} \leqslant \text { const. }\left\|\phi_{1}\right\|_{L^{2}}\left\|\phi_{2}\right\|_{H^{1}} .
$$

Therefore,

$$
\|\mathscr{F}\|_{L^{2}} \leqslant \text { const. }\left\|\phi_{1}\right\|_{L^{2}}\left\|\phi_{2}\right\|_{H^{1}}\left\|\tilde{\phi}_{2}\right\|_{L^{2}} .
$$

The modulus of (3.2) satisfies by similar arguments

$$
\begin{aligned}
|\mathscr{J}| & \leqslant \text { const. }\left|\left\langle\phi_{1}(x) \tilde{\phi}_{1}(y)\left|\frac{1}{|x-y|}\right| \phi_{2}(x) \tilde{\phi}_{2}(y)\right\rangle_{L^{2}(x, y)}\right| \\
& \leqslant \text { const. }\left\|\left\langle\phi_{1}(x)\left|\frac{1}{|x-y|}\right| \phi_{2}(x)\right\rangle_{L^{2}(x)}\right\|_{L^{\infty}(y)}\left|\left\langle\tilde{\phi}_{1} \mid \tilde{\phi}_{2}\right\rangle_{L^{2}}\right| \\
& \leqslant \text { const. }\left\|\phi_{1}\right\|_{L^{2}}\left\|\phi_{2}\right\|_{H^{1}}\left\|\tilde{\phi}_{1}\right\|_{L^{2}}\left\|\tilde{\phi}_{2}\right\|_{L^{2}} .
\end{aligned}
$$

Next, we consider the $H^{1}$-norm of $\mathscr{F}$. Noting that

$$
\nabla_{y} \frac{1}{|x-y|}=\frac{1}{|x-y|^{2}} \vec{e}_{x-y}
$$


where $\vec{e}_{x-y}$ denotes the unit vector in direction $x-y$, we find similarly as in (3.10)

$$
\begin{aligned}
\|\nabla \mathscr{F}\|_{L^{2}} \leqslant & \|\| \frac{1}{|x-y|} \phi_{1}(x)\left\|_{L^{2}(x)}\right\|\left\|_{L^{\infty}(y)}\right\|\left\|\frac{1}{|x-y|} \phi_{2}(x)\right\|_{L^{2}(x)}\left\|_{L^{\infty}(y)}\right\| \tilde{\phi}_{2} \|_{L^{2}} \\
& + \text { const. }\left\|\phi_{1}\right\|_{L^{2}}\left\|\phi_{2}\right\|_{H^{1}}\left\|\tilde{\phi}_{2}\right\|_{H^{1}} \\
\leqslant & \text { const. }\left(\left\|\phi_{1}\right\|_{H^{1}}\left\|\phi_{2}\right\|_{H^{1}}\left\|\tilde{\phi}_{2}\right\|_{L^{2}}+\left\|\phi_{1}\right\|_{L^{2}}\left\|\phi_{2}\right\|_{H^{1}}\left\|\tilde{\phi}_{2}\right\|_{H^{1}}\right) .
\end{aligned}
$$

Next, we use the fact that $(4 \pi|x|)^{-1}$ is the fundamental solution of the three-dimensional Laplacian and the bound $\left\|\bar{\phi}_{1} \phi_{2} \tilde{\phi}_{2}\right\|_{L^{2}} \leqslant$ const. $\left\|\phi_{1}\right\|_{H^{1}}\left\|\phi_{2}\right\|_{H^{1}}\left\|\tilde{\phi}_{2}\right\|_{H^{1}}$ (which follows from the Sobolev embedding $H^{1} \subset L^{6}$ ) together with the previous estimates to obtain (3.7) (this estimate is also given in [6, p.976]). Note in particular that the bound depends only linearly on the $H^{2}$-norms of the $\phi$ 's.

To verify that $\mathscr{F}$ is locally Lipschitz from $H^{2} \rightarrow H^{2}$, the same estimates as above are used, where one of the $\phi$ 's is replaced by the difference between two orbitals, and the same is done for $\mathscr{J}$.

The outline of the proof of Theorem 3.1 is similar to [7]: first it is shown that solutions in $H^{2}$ exist locally in time, where the growth in the $H^{2}$ norm is exponential in terms of a bound of the $H^{1}$ norm of the solution, and also a bound of the inverse $\rho^{-1}$ of the density matrix. Since the $H^{1}$ norm of a strong solution can be bounded by the constant total energy, it follows that the $H^{1}$ norm remains in fact bounded and the $H^{2}$ norm grows at worst exponentially as long as $\rho^{-1}$ remains bounded. There are, however, some subtleties that need to be addressed carefully.

We write the system (2.8)-(2.9) compactly as

$$
\begin{aligned}
\mathrm{i} \frac{d a}{d t} & =A_{V}(\phi) a, \\
\mathrm{i} \frac{\partial \phi}{\partial t} & =T \phi+B_{V}(a, \phi) \phi .
\end{aligned}
$$

We will use the following norms: For coefficient vectors $a=\left(a_{J}\right) \in \mathbb{C}^{D}$, where $D=\left(\begin{array}{l}N \\ d\end{array}\right)$, we use the Euclidean norm $\|a\|=\left(\sum_{J}\left|a_{J}\right|^{2}\right)^{1 / 2}$. For the orbitals $\phi=\left(\phi_{j}\right) \in\left(L^{2}\right)^{N}$ we use $\|\phi\|_{S}=\max _{j}\left\|\phi_{j}\right\|_{S}$, where $\left\|\phi_{j}\right\|_{S}$ denotes the norm in the spaces $S=L^{2}, H^{1}, H^{2}$ etc. For the pair $z=(a, \phi)$, we use the norm $\|(a, \phi)\|_{S}=\max \left\{\|a\|,\|\phi\|_{S}\right\}$.

Theorem 3.1 is proven by verifying the assumptions of the existence result from [28] (also restated in [26, Section 6.1]) for an abstract initial value problem on the Banach space $X$,

$$
\frac{d z}{d t}(t)+A z(t)=f(t, z(t)), \quad z(0)=z^{0} \in X .
$$

If $f:[0, \infty) \times X \rightarrow X$ is continuous in $t$ for $t \geqslant 0$ and locally Lipschitz in $z$, uniformly in $t$ on bounded intervals, and $-A$ is the infinitesimal generator of a strongly continuous semigroup on $X$, then for every $z^{0} \in X$ there is a $t^{*}>0$ such that the abstract initial value problem (3.17) has a unique strong solution $z$ on $\left[0, t^{*}\right)$. Moreover, if $t^{*}<\infty$, then $\lim _{t \rightarrow t^{*}}\|z(t)\|=\infty$.

Proof of Theorem 3.1: In order to prove that the system (2.8)-(2.9) with initial data $\left(a^{0}, \phi^{0}\right)$ has a unique solution in $H^{2}$ at least for small $t$, we show that this system satisfies the assumptions of the above existence result for abstract initial value problems. Subsequently, we will show that the solution remains bounded in $H^{2}$ as long as $\rho$ is nonsingular.

We need to consider the dependence of $A_{V}$ and $B_{V}$ on the coefficient vector $a$ and the orbitals $\phi$. From the smooth dependence of $\rho^{-1}$ on $a$ when $\rho$ is nonsingular, and the estimates for $\mathscr{F}$ and $\mathscr{J}$ 
above, we obtain that estimates

$$
\left\|\left(A_{V}(\phi) a, B_{V}(a, \phi) \phi\right)-\left(A_{V}(\tilde{\phi}) \tilde{a}, B_{V}(\tilde{a}, \tilde{\phi}) \tilde{\phi}\right)\right\|_{S} \leqslant C_{1}\|(a, \phi)-(\tilde{a}, \tilde{\phi})\|_{S},
$$

hold locally in the spaces $S=L^{2}, H^{1}, H^{2}$, with a constant $C_{1}$ depending on the $H^{1}$-norms of $\phi$ and $\tilde{\phi}$ and bounds of $a$ and $\rho^{-1}$.

With the existence result for abstract initial value problems applied to the system (2.8)-(2.9), we now conclude that a strong solution $\phi$ exists in $H^{2}$ on some interval $\left[0, t_{1}\right]$ with $t_{1}>0$. This further implies that $\phi_{j}$ satisfy the orthonormality relations (2.6), provided that the initial data are orthonormal; see [18]. We then conclude that $u=\sum_{J} a_{J} \Phi_{J}$ indeed solves the variational problem (2.5).

Having come this far, we note next that the $L^{2}$ and the $H^{1}$ norms of $u$ are bounded along solutions of (2.5): The $L^{2}$-norm is conserved, which follows immediately on taking the real part in (2.7). Moreover, the total energy $E:=\langle u|\mathbf{H}| u\rangle$ of variational approximations is conserved; see, e.g., [2] or [22, Section II.1]. Hence, the trivial bound of the kinetic energy operator $\mathbf{T}$,

$$
\frac{1}{2}\|u\|_{H^{1}}^{2}=\langle u|\mathbf{T}| u\rangle \leqslant E
$$

implies bounded $H^{1}$-norm of $u$. Now, a calculation using the orthogonality relation (2.6) and formula (2.11) shows that

$$
\left\|\partial_{i}^{(k)} u\right\|_{L^{2}}^{2}=\sum_{j, l} \rho_{j l}\left\langle\partial_{i} \phi_{j} \mid \partial_{i} \phi_{l}\right\rangle
$$

where $\partial_{i}^{(k)}$ is the partial derivative with respect to the $i$-th component of the position variable $x^{(k)}$ of the $k$-th particle, and $\partial_{i}$ is the partial derivative with respect to the $i$-th component of $x$. Since $\rho$ is hermitian positive definite, it follows that the $H^{1}$ norm of $\phi$ can be bounded in terms of the $H^{1}$ norm of $u$, with a constant that depends on a bound of $\rho^{-1}$.

To derive a bound for the $H^{2}$-norm, we apply $T=-\frac{1}{2} \Delta$ to the integrated form of the differential equation for $\phi$,

$$
\phi(t)=\mathrm{e}^{-\mathrm{i} t T} \phi_{0}-\mathrm{i} \int_{0}^{t} \mathrm{e}^{-\mathrm{i}(t-s) T} B_{V}(a(s), \phi(s)) d s .
$$

With the estimate (3.7), we find that

$$
\|T \phi(t)\|_{L^{2}} \leqslant\left\|T \phi_{0}\right\|_{L^{2}}+C \int_{0}^{t}\|\phi(\tau)\|_{H^{2}} d \tau
$$

with a constant $C$ that depends only on bounds of $\rho^{-1}$ and of the $L^{2}$ - and $H^{1}$-norms of $\phi$ on the considered time interval. Since $\|\phi(t)\|_{H^{2}} \sim\|\phi(t)\|_{L^{2}}+\|T \phi(t)\|_{L^{2}}$, we obtain with the Gronwall lemma that

$$
\|\phi(t)\|_{H^{2}} \leqslant \text { const. }\|\phi(0)\|_{H^{2}} e^{C t} .
$$

Since the $L^{2}$ - and $H^{1}$-norms of $\phi$ have been shown to be bounded, we conclude overall that $\phi$ can grow at worst exponentially in $t$ on any interval on which a bound of $\rho^{-1}$ is available. In conjunction with the existence result for abstract initial value problems on page 6, this shows the assertion of Theorem 3.1.

REMARK 3.1 On rewriting (3.1) as

$$
\mathscr{F}(y)=\left\langle\phi_{1}(y-x)\left|\frac{1}{|x|}\right| \phi_{2}(y-x)\right\rangle_{L^{2}(x)} \tilde{\phi}_{2}(y)
$$


and differentiating the differential equations for the $\phi_{j}$, the above result is readily extended to show that for every $m \geqslant 2$, the orbitals $\phi_{j}$ are in $H^{m}$ if the initial data is in $H^{m}$.

REMARK 3.2 For the extension to the more elaborate single-particle operators (1.3), we note that (1.3) still generates a strongly continuous semigroup on $H^{2}$, since $1 /|x| \ll \Delta[12]$. Thus the proposition remains valid with the same proof.

\section{Analysis of Variational Splitting}

\subsection{Variational Splitting}

The variational splitting method for the time integration of a Schrödinger equation with Hamiltonian $\mathbf{H}=\mathbf{T}+\mathbf{V}$ reduced to an approximation manifold $\mathscr{M}$ by the Dirac-Frenkel time-dependent variational principle (2.5) was introduced in [20]. One step $u^{0} \mapsto u^{1}=\mathbf{S}_{\Delta t}\left(u^{0}\right)$ of the method with step size $\Delta t$, starting at $u^{0} \in \mathscr{M}$, is defined as follows:

- Compute $u_{-}^{1 / 2} \in \mathscr{M}$ as the solution at time $t_{0}+\frac{1}{2} \Delta t$ of

$$
\left\langle\delta u \mid \mathrm{i} \frac{\partial u}{\partial t}-\mathbf{T} u\right\rangle=0 \quad \forall \delta u \in \mathscr{T}_{u} \mathscr{M},
$$

with initial value $u\left(t_{0}\right)=u^{0}$.

- Compute $u_{+}^{1 / 2} \in \mathscr{M}$ as the solution at time $t_{0}+\Delta t$ of

$$
\left\langle\delta u \mid \mathrm{i} \frac{\partial u}{\partial t}-\mathbf{V} u\right\rangle=0 \quad \forall \delta u \in \mathscr{T}_{u} \mathscr{M},
$$

with initial value $u\left(t_{0}\right)=u_{-}^{1 / 2}$.

- Compute $u^{1} \in \mathscr{M}$ as the solution at time $t_{0}+\Delta t$ of (4.1) with initial value $u\left(t_{0}+\frac{1}{2} \Delta t\right)=u_{+}^{1 / 2}$.

For the MCTDHF approximation with the gauge (2.7), this is equivalent to using the usual second order, symmetric operator splitting (commonly known as Strang splitting) on the MCTDHF equations (2.8)(2.9) $[16,20]$. Since for MCTDHF we have $\mathbf{T} u \in \mathscr{T}_{u} \mathscr{M}$ for $u \in \mathscr{M} \cap H^{2}$, the two steps (4.1) just solve the free Schrödinger equation

$$
\mathrm{i} \frac{\partial u}{\partial t}=\mathbf{T} u
$$

For initial data in $\mathscr{M}$, this decouples into single-particle free Schrödinger equations:

$$
\begin{aligned}
& \frac{d a_{J}}{d t}=0, \quad \forall J, \\
& \mathrm{i} \frac{\partial \phi_{j}}{\partial t}=T \phi_{j}, \quad j=1, \ldots, N .
\end{aligned}
$$

The step (4.2) amounts to the solution of the nonlinear system, cf. (3.15)-(3.16),

$$
\mathrm{i} \frac{d a}{d t}=A_{V}(\phi) a, \quad \mathrm{i} \frac{\partial \phi}{\partial t}=B_{V}(a, \phi) \phi,
$$


which in practice is solved approximately with one or a few steps of an explicit time-stepping method followed by occasional reorthogonalization of the orbitals. Since the Laplacian has been taken out of the equation, there is no time-step restriction $\Delta t=O\left(\Delta x^{2}\right)$ as for a direct treatment of (3.16). Advantages of this splitting have been described in [15] and [20], for instance. Convergence properties for bounded potentials have been studied in [20]. For Schrödinger-Poisson equations, the symmetric splitting method was analyzed in [21]. The error analysis of the present paper uses similar techniques.

\subsection{Error Bounds for the Variational Splitting}

We will prove the following convergence result.

THEOREM 4.1 Consider the approximation of (2.8)-(2.9) by semi-discretization in time with the variational splitting method $u^{n+1}=\mathbf{S}_{\Delta t}\left(u^{n}\right), n=0,1, \ldots$ Then, the convergence estimates

$$
\begin{aligned}
\left\|u^{n}-u\left(t_{n}\right)\right\|_{H^{1}} & \leqslant C \Delta t \\
\left\|u^{n}-u\left(t_{n}\right)\right\|_{L^{2}} & \leqslant C \Delta t^{2},
\end{aligned}
$$

hold uniformly for $0 \leqslant t_{n}=n \Delta t \leqslant \bar{t}$ if the MCTDHF solution $u(t)$ is in $H^{2}$. The constant $C$ depends on the maximum of the $H^{2}$ norm of $u(t)$ for $0 \leqslant t \leqslant \bar{t}$.

\subsection{Outline of the Convergence Proof for Variational Splitting}

We denote by $u(t) \in \mathscr{M}$ the MCTDHF wave function with coefficients and orbitals $(a(t), \phi(t))$ that are the exact solution of the MCTDHF equations (2.8)-(2.9), and by $u^{n} \in \mathscr{M}$ the approximate discrete-time wave function with coefficients and orbitals $\left(a^{n}, \phi^{n}\right)$ resulting from the variational splitting, which we denote by

$$
\left(a^{n+1}, \phi^{n+1}\right)=S_{\Delta t}\left(a^{n}, \phi^{n}\right) .
$$

Since this amounts to a symmetric splitting of the vector field defining (2.8)-(2.9), we set

$$
\hat{T}(a, \phi):=-\mathrm{i}\left(\begin{array}{c}
0 \\
T \phi
\end{array}\right), \quad \hat{V}(a, \phi):=-\mathrm{i}\left(\begin{array}{c}
A_{V}(\phi) a \\
B_{V}(a, \phi) \phi
\end{array}\right), \quad \hat{H}:=\hat{T}+\hat{V} .
$$

In our estimates we will work with orbitals $\phi \in\left(H^{2}\right)^{N}$ and coefficients $a \in \mathbb{C}^{D}$ rather than with the corresponding wave function $u \in \mathscr{M}$, for which the theorem is formulated. However, in view of the identity (cf. (3.19))

$$
\left\|\left(\partial_{i}^{(k)}\right)^{m} u\right\|_{L^{2}}^{2}=\sum_{j, l} \rho_{j l}\left\langle\partial_{i}^{m} \phi_{j} \mid \partial_{i}^{m} \phi_{l}\right\rangle
$$

we can switch back and forth between Sobolev-norm estimates of $u$ and $\phi$ on any interval where the hermitian positive definite density matrix $\rho$ has a bounded inverse. For $(a, \phi)$ we use the norms given after (3.15)-(3.16).

Step 1 First, stability in the $H^{1}$ norm is shown: If $a, \tilde{a} \in \mathbb{C}^{D}$ are of unit norm and $\phi, \tilde{\phi} \in\left(H^{1}\right)^{N}$ with

$$
\|\phi\|_{H^{1}} \leqslant M_{1}, \quad\|\tilde{\phi}\|_{H^{1}} \leqslant M_{1}
$$

then we have

$$
\left\|S_{\Delta t}(a, \phi)-S_{\Delta t}(\tilde{a}, \tilde{\phi})\right\|_{H^{1}} \leqslant \mathrm{e}^{c_{1} \Delta t}\|(a, \phi)-(\tilde{a}, \tilde{\phi})\|_{H^{1}},
$$

with a constant $c_{1}=c_{1}\left(M_{1}\right)$. 
Step 2 We then estimate the local error in $H^{1}$. If

$$
\|\phi(t)\|_{H^{2}} \leqslant M_{2} \quad \text { for } 0 \leqslant t \leqslant \bar{t},
$$

then, for $0 \leqslant t \leqslant \bar{t}-\Delta t$,

$$
\left\|S_{\Delta t}(a(t), \phi(t))-(a(t+\Delta t), \phi(t+\Delta t))\right\|_{H^{1}} \leqslant c_{2} \Delta t^{2},
$$

with a constant $c_{2}=c_{2}\left(M_{2}\right)$. In this argument, a bound for the Lie commutator $\|[\hat{T}, \hat{V}](a, \phi)\|_{H^{1}}$ is used. This depends on the $H^{2}$-norm of $\phi$.

Step 3 Combining stability (4.12) and consistency (4.14) in $H^{1}$, the standard argument referred to as Lady Windermere's fan in [11, Section II.3] then yields convergence in $H^{1}$,

$$
\left\|\left(a^{n}, \phi^{n}\right)-\left(a\left(t_{n}\right), \phi\left(t_{n}\right)\right)\right\|_{H^{1}} \leqslant C_{2} \Delta t, \quad \text { for } t_{n}=n \Delta t \leqslant \bar{t}, \quad C_{2}=C_{2}\left(M_{2}\right),
$$

which further yields (4.7), and in particular boundedness of the numerical solution in $H^{1}$,

$$
\left\|\phi^{n}\right\|_{H^{1}} \leqslant \text { const. }
$$

Step 4 Next, $H^{1}$-conditional stability in $L^{2}$ is shown: If $a, \tilde{a} \in \mathbb{C}^{D}$ are of unit norm and $\phi, \tilde{\phi} \in\left(H^{1}\right)^{N}$ are bounded in the $H^{1}$ norm by $M_{1}$, then

$$
\left\|S_{\Delta t}(a, \phi)-S_{\Delta t}(\tilde{a}, \tilde{\phi})\right\|_{L^{2}} \leqslant \mathrm{e}^{c_{1} \Delta t}\|(a, \phi)-(\tilde{a}, \tilde{\phi})\|_{L^{2}},
$$

with a constant $c_{1}=c_{1}\left(M_{1}\right)$.

Step 5 Then, the local error in $L^{2}$ is estimated. To this end, the $L^{2}$-norm of the double commutator $\|[\hat{T},[\hat{T}, \hat{V}]](a, \phi)\|_{L^{2}}$ is estimated. The bound depends on $M_{2}$ from (4.13). From this it is concluded that, for $0 \leqslant t \leqslant \bar{t}-\Delta t$,

$$
\left\|S_{\Delta t}(a(t), \phi(t))-(a(t+\Delta t), \phi(t+\Delta t))\right\|_{L^{2}} \leqslant c_{2} \Delta t^{3},
$$

where $c_{2}=c_{2}\left(M_{2}\right)$.

Step 6 Since we previously concluded in (4.16) that $\left\|\phi^{n}\right\|_{H^{1}}$ is bounded, the stability estimate (4.17) in conjunction with (4.18) now yields second-order convergence in $L^{2}$ :

$$
\left\|\left(a^{n}, \phi^{n}\right)-\left(a\left(t_{n}\right), \phi\left(t_{n}\right)\right)\right\|_{L^{2}} \leqslant C_{2} \Delta t^{2} \quad \text { for } t_{n}=n \Delta t \leqslant \bar{t}, \quad C_{2}=C_{2}\left(M_{2}\right),
$$

which further yields (4.8).

\subsection{Calculus of Lie Derivatives}

For the convergence analysis, a convenient notation to derive estimates for the local error of the splitting method is given by the calculus of Lie derivatives; see also [10], [21].

For a (nonlinear) vector field $F$, such as $\hat{H}$ or $\hat{T}$ or $\hat{V}$, we denote by $\varphi_{F}^{t}$ the corresponding flow, so that $\varphi_{F}^{t}\left(z_{0}\right)$ is the solution at time $t$ of the differential equation $\dot{z}=F(z), z(0)=z_{0}$. If $G$ is another vector field, the Lie derivative $D_{F}$ is defined by

$$
\left(D_{F} G\right)(z)=\left.\frac{d}{d t}\right|_{t=0} G\left(\varphi_{F}^{t}(z)\right)=G^{\prime}(z) F(z) .
$$


We now define

$$
\left(\exp \left(t D_{F}\right) G\right)(z):=G\left(\varphi_{F}^{t}(z)\right) .
$$

If $G$ is the identity, the exact flow is thus reproduced as $\exp \left(t D_{F}\right) \operatorname{Id}(z)=\varphi_{F}^{t}(z)$. We have the following manipulation rule:

$$
\frac{d}{d t} \exp \left(t D_{F}\right) G(z)=\left(D_{F} \exp \left(t D_{F}\right) G\right)(z)=\left(\exp \left(t D_{F}\right) D_{F} G\right)(z)
$$

Moreover, we will use that the commutator $\left[D_{F}, D_{G}\right]=D_{F} D_{G}-D_{G} D_{F}$ of the Lie derivatives of two vector fields $F$ and $G$ is the Lie derivative of the commutator $[G, F]=G^{\prime} F-F^{\prime} G$ of the vector fields in reversed order:

$$
\left[D_{F}, D_{G}\right]=D_{[G, F]}
$$

\subsection{Commutator bounds}

In the estimation of the local errors of the variational splitting (Steps 2 and 5) we need to derive bounds for commutators of the vector fields $\hat{T}$ and $\hat{V}$. We find that commutators of the nonlinear parts of (2.8)(2.9) with the kinetic energy operators contain terms of the following forms, after taking into account the antisymmetry expressed in (3.3):

$$
\begin{aligned}
& \mathscr{C}_{1}(y):=\left\langle\phi_{1}(x)\left|\nabla_{y} \frac{1}{|x-y|}\right| \phi_{2}(x)\right\rangle_{L^{2}(x)} \nabla_{y} \phi_{3}(y), \\
& \mathscr{C}_{2}(y):=\left\langle\phi_{1}(x)\left|\nabla_{x} \frac{1}{|x-y|}\right| \nabla_{x} \phi_{2}(x)\right\rangle_{L^{2}(x)} \phi_{3}(y) .
\end{aligned}
$$

These terms can be estimated as in Lemma 3.1. We obtain the following bounds:

$$
\begin{aligned}
\left\|\mathscr{C}_{1}\right\|_{H^{1}} & \leqslant \text { const. }\left\|\phi_{1}\right\|_{H^{1}}\left\|\phi_{2}\right\|_{H^{1}}\left\|\phi_{3}\right\|_{H^{2}}, \\
\left\|\mathscr{C}_{2}\right\|_{H^{1}} & \leqslant \text { const. }\left\|\phi_{1}\right\|_{H^{1}}\left\|\phi_{2}\right\|_{H^{2}}\left\|\phi_{3}\right\|_{H^{1}} .
\end{aligned}
$$

Repeating the analogous derivations to compute the terms in the double commutator $[\hat{T},[\hat{T}, \hat{V}]]$ reveals that the only terms which do not cancel due to antisymmetry, take the form

$$
\begin{aligned}
\mathscr{C}_{3}(y) & :=\left\langle\phi_{1}(x)\left|\nabla_{x} \Delta_{y} \frac{1}{|x-y|}\right| \nabla_{x} \phi_{2}(x)\right\rangle_{L^{2}(x)} \phi_{3}(y) \\
& =\left(\nabla_{y} \overline{\phi_{1}(y)} \cdot \nabla_{y} \phi_{2}(y)+\overline{\phi_{1}(y)} \Delta \phi_{2}(y)\right) \phi_{3}(y),
\end{aligned}
$$

which is bounded by

$$
\left\|\mathscr{C}_{3}\right\|_{L^{2}} \leqslant \text { const. }\left\|\phi_{1}\right\|_{H^{2}}\left\|\phi_{2}\right\|_{H^{2}}\left\|\phi_{3}\right\|_{H^{2}}
$$

on using the Hölder inequality and the Sobolev embeddings $H^{1} \subset L^{4}$ and $L^{\infty} \subset H^{2}$.

\subsection{Proof details}

We now work out the details of the roadmap given in Section 4.3, using the estimates from Sections 3 and 4.5. Without restriction of generality, we let $t_{0}=0$. 
Step 1 The substeps (4.1) amount to the solution of free Schrödinger equations (4.3), which preserve the $H^{1}$-norm. The substep (4.2) with the potential requires the solution of (4.6). Since the right-hand side is locally $H^{1}$-Lipschitz by (3.18), the $H^{1}$ stability bound (4.12) follows with the Gronwall lemma.

Step 2 We denote by $D_{H}, D_{T}$ and $D_{V}$ the Lie derivatives defined by the vector fields $\hat{H}, \hat{T}$ and $\hat{V}$, respectively, see (4.9), omitting for simplicity the hats in the subscripts. For a shorter notation, we write for the vector containing the coefficients and the orbitals $z:=(a, \phi)$. We recast the exact solution $z(t)=\exp \left(t D_{H}\right) \operatorname{Id}\left(z^{0}\right)$ using the nonlinear variation-of-constants formula (also known as Gröbner-Alekseev formula [11, p. 98]) twice,

$$
\begin{aligned}
z(t) & =\exp \left(t D_{H}\right) \operatorname{Id}\left(z^{0}\right) \\
& =\exp \left(t D_{T}\right) \operatorname{Id}\left(z^{0}\right)+\int_{0}^{t} \exp \left((t-s) D_{H}\right) D_{V} \exp \left(s D_{T}\right) \operatorname{Id}\left(z^{0}\right) d s \\
& =\exp \left(t D_{T}\right) \operatorname{Id}\left(z^{0}\right)+\int_{0}^{t} \exp \left((t-s) D_{T}\right) D_{V} \exp \left(s D_{T}\right) \operatorname{Id}\left(z^{0}\right) d s+r_{1}
\end{aligned}
$$

with the remainder term given by

$$
r_{1}=\int_{0}^{t} \int_{0}^{t-s} \exp \left((t-s-\sigma) D_{H}\right) D_{V} \exp \left(\sigma D_{T}\right) D_{V} \exp \left(s D_{T}\right) \operatorname{Id}\left(z^{0}\right) d \sigma d s .
$$

On the other hand, we observe that a step of the numerical method can be recast as

$$
z^{1}=S_{\Delta t}\left(z^{0}\right)=\exp \left(\frac{1}{2} \Delta t D_{T}\right) \exp \left(\Delta t D_{V}\right) \exp \left(\frac{1}{2} \Delta t D_{T}\right) \operatorname{Id}\left(z^{0}\right)
$$

whence by Taylor expansion of $\exp \left(\Delta t D_{V}\right)$

$$
z^{1}=\exp \left(\Delta t D_{T}\right) \operatorname{Id}\left(z^{0}\right)+\Delta t \exp \left(\frac{1}{2} \Delta t D_{T}\right) D_{V} \exp \left(\frac{1}{2} \Delta t D_{T}\right) \operatorname{Id}\left(z^{0}\right)+r_{2}
$$

with

$$
r_{2}=\Delta t^{2} \int_{0}^{1}(1-\theta) \exp \left(\frac{1}{2} \Delta t D_{T}\right) \exp \left(\theta \Delta t D_{V}\right) D_{V}^{2} \exp \left(\frac{1}{2} \Delta t D_{T}\right) \operatorname{Id}\left(z^{0}\right) d \theta .
$$

Subtracting (4.30) with $t=\Delta t$ from (4.33), we thus obtain

$$
\begin{aligned}
z^{1}-z(\Delta t)= & \Delta t \exp \left(\frac{1}{2} \Delta t D_{T}\right) D_{V} \exp \left(\frac{1}{2} \Delta t D_{T}\right) \operatorname{Id}\left(z^{0}\right) \\
& -\int_{0}^{\Delta t} \exp \left((\Delta t-s) D_{T}\right) D_{V} \exp \left(s D_{T}\right) \operatorname{Id}\left(z^{0}\right) d s+r_{2}-r_{1}
\end{aligned}
$$

Thus, the principal error term corresponds with the quadrature error of the midpoint rule applied to the integral over $[0, \Delta t]$ of the function

$$
f(s):=\exp \left((\Delta t-s) D_{T}\right) D_{V} \exp \left(s D_{T}\right) \operatorname{Id}\left(z^{0}\right) .
$$


The quadrature error can be written in first order Peano form as

$$
\Delta t f(\Delta t / 2)-\int_{0}^{\Delta t} f(s) d s=\Delta t^{2} \int_{0}^{1} \kappa_{1}(\sigma) f^{\prime}(\sigma \Delta t) d \sigma
$$

with the scalar, bounded Peano kernel $\kappa_{1}$ of the midpoint rule. We can now compute

$$
\begin{aligned}
f^{\prime}(s) & =-\exp \left((\Delta t-s) D_{T}\right)\left[D_{T}, D_{V}\right] \exp \left(s D_{T}\right) \operatorname{Id}\left(z^{0}\right) \\
& =\exp \left((\Delta t-s) D_{T}\right) D_{[\hat{T}, \hat{V}]} \exp \left(s D_{T}\right) \operatorname{Id}\left(z^{0}\right) \\
& =\exp (s \hat{T})[\hat{T}, \hat{V}]\left(\exp ((\Delta t-s) \hat{T}) z^{0}\right) .
\end{aligned}
$$

By virtue of the commutator bounds derived in Section 4.5 and the unitarity of $\exp (s \hat{T})=$ $\operatorname{blockdiag}\left(1, e^{-i s T}\right)$, it follows that

$$
\left\|\Delta t f(\Delta t / 2)-\int_{0}^{\Delta t} f(s) d s\right\|_{H^{1}} \leqslant c_{2} \Delta t^{2}
$$

where $c_{2}$ depends on the $H^{2}$ norm of $z$. It remains to derive a bound for $\left\|r_{2}-r_{1}\right\|_{H^{1}} \leqslant\left\|r_{1}\right\|_{H^{1}}+$ $\left\|r_{2}\right\|_{H^{1}}$. Setting $\tau:=\Delta t-s-\sigma$, we observe that

$$
\begin{aligned}
& \exp \left(\tau D_{H}\right) D_{V} \exp \left(\sigma D_{T}\right) D_{V} \exp \left(s D_{T}\right) \operatorname{Id}\left(z^{0}\right) \\
& \quad=\exp (s \hat{T}) \hat{V}^{\prime}(\exp (\sigma \hat{T}) z(\tau)) \exp (\sigma \hat{T}) \hat{V}(z(\tau)) \\
& \exp \left(\frac{\Delta t}{2} D_{T}\right) \exp \left(\theta \Delta t D_{V}\right) D_{V}^{2} \exp \left(\frac{\Delta t}{2} D_{T}\right) \operatorname{Id}\left(z^{0}\right)=\exp \left(\frac{\Delta t}{2} \hat{T}\right) \hat{V}^{\prime}(\zeta) \hat{V}(\zeta), \\
& \quad \text { where } \quad \zeta:=\varphi_{V}^{\theta \Delta t}\left(z_{-}^{1 / 2}\right), \quad z_{-}^{1 / 2}:=\exp \left(\frac{\Delta t}{2} \hat{T}\right) z^{0} .
\end{aligned}
$$

Analogously to the proof of (4.12) (see Step 1 of this proof) we conclude that

$$
\|\zeta\|_{H^{1}} \leqslant \exp \left(c_{1} \Delta t\right)\left\|z^{0}\right\|_{H^{1}}
$$

Finally, with the bounds

$$
\|\hat{V}(z)\|_{H^{1}} \leqslant C_{1}, \quad\left\|\hat{V}^{\prime}(z) w\right\|_{H^{1}} \leqslant C_{1}\|w\|_{H^{1}},
$$

with a constant $C_{1}$ depending on the $H^{1}$-norm of $z$, we conclude

$$
\left\|r_{1}-r_{2}\right\|_{H^{1}} \leqslant\left\|r_{1}\right\|_{H^{1}}+\left\|r_{2}\right\|_{H^{1}} \leqslant C \Delta t^{2}
$$

with another constant depending on the $H^{1}$-norm of $z$.

Step 3 This follows directly from Lady Windermere's fan [11].

Step 4 This is concluded analogously as Step 1, noting (3.5) and (3.8).

Step 5 We revisit the error representation (4.34). We now write the principal error term in second order Peano form

$$
\Delta t f(\Delta t / 2)-\int_{0}^{\Delta t} f(s) d s=\Delta t^{3} \int_{0}^{1} \kappa_{2}(\sigma) f^{\prime \prime}(\sigma \Delta t) d \sigma
$$


with the scalar, bounded Peano kernel $\kappa_{2}$ of the midpoint rule. Rearranging, it follows

$$
\begin{aligned}
f^{\prime \prime}(s) & =-\exp \left((\Delta t-s) D_{T}\right)\left[D_{T},\left[D_{T}, D_{V}\right]\right] \exp \left(s D_{T}\right) \operatorname{Id}\left(z^{0}\right) \\
& =\exp \left((\Delta t-s) D_{T}\right) D_{[\hat{T},[\hat{T}, \hat{V}]]} \exp \left(s D_{T}\right) \operatorname{Id}\left(z^{0}\right) \\
& =\exp (s \hat{T})[\hat{T},[\hat{T}, \hat{V}]]\left(\exp ((\Delta t-s) \hat{T}) z^{0}\right) .
\end{aligned}
$$

Thus, by the estimates derived in Section 4.5, it is clear that

$$
\left\|\Delta t f(\Delta t / 2)-\int_{0}^{\Delta t} f(s) d s\right\|_{L^{2}} \leqslant c_{2} \Delta t^{3}
$$

where $c_{2}$ depends on $\|z\|_{H^{2}}$. Finally, we estimate the remainder terms. Defining

$$
g(s, \sigma):=\exp \left((\Delta t-s-\sigma) D_{T}\right) D_{V} \exp \left(\sigma D_{T}\right) D_{V} \exp \left(s D_{T}\right) \operatorname{Id}\left(z^{0}\right),
$$

we can write

$$
r_{2}-r_{1}=\frac{\Delta t^{2}}{2} g(\Delta t / 2,0)-\int_{0}^{\Delta t} \int_{0}^{\Delta t-s} g(s, \sigma) d \sigma d s+\tilde{r}_{2}-\tilde{r}_{1} .
$$

Similarly as in Step 2, the remainders satisfy

$$
\left\|\tilde{r}_{1}\right\|_{L^{2}}+\left\|\tilde{r}_{2}\right\|_{L^{2}} \leqslant C_{1} \Delta t^{3},
$$

where $C_{1}$ depends on $\|z\|_{H^{1}}$. The other terms in (4.42) represent the local error of a first-order, two-dimensional quadrature formula, and hence

$$
\begin{aligned}
& \left\|\frac{\Delta t^{2}}{2} g(\Delta t / 2,0)-\int_{0}^{\Delta t} \int_{0}^{\Delta t-s} g(s, \sigma) d \sigma d s\right\|_{L^{2}} \\
& \quad \leqslant \text { const. } \Delta t^{3}\left(\max \left\|\frac{\partial g}{\partial s}\right\|_{L^{2}}+\max \left\|\frac{\partial g}{\partial \sigma}\right\|_{L^{2}}\right),
\end{aligned}
$$

with the maxima taken over the triangle defined by $0 \leqslant s \leqslant \Delta t, 0 \leqslant \sigma \leqslant \Delta t-s$. Computing

$$
\begin{aligned}
\frac{\partial g}{\partial s}(s, \sigma)= & \exp \left((\Delta t-s-\sigma) D_{T}\right) D_{[\hat{T}, \hat{V}]} \exp \left(\sigma D_{T}\right) D_{V} \exp \left(s D_{T}\right) \operatorname{Id}\left(z^{0}\right) \\
& +\exp \left((\Delta t-s-\sigma) D_{T}\right) D_{V} \exp \left(\sigma D_{T}\right) D_{[\hat{T}, \hat{V}]} \exp \left(s D_{T}\right) \operatorname{Id}\left(z^{0}\right) \\
\frac{\partial g}{\partial \sigma}(s, \sigma)= & \exp \left((\Delta t-s-\sigma) D_{T}\right) D_{[\hat{T}, \hat{V}]} \exp \left(\sigma D_{T}\right) D_{V} \exp \left(s D_{T}\right) \operatorname{Id}\left(z^{0}\right)
\end{aligned}
$$

we realize that these partial derivatives only contain $\hat{V}$ and $[\hat{T}, \hat{V}]$ together with their derivatives, whose $L^{2}$-norms can be estimated in terms of $\|z\|_{H^{2}}$ by virtue of (4.38). Altogether, we obtain

$$
\left\|r_{2}-r_{1}\right\|_{L^{2}} \leqslant C \Delta t^{3}
$$

where the constant $C$ depends on $\|z\|_{H^{2}}$. Recalling (4.34), we obtain (4.18).

Step 6 Uses the same standard argument as in Step 3. 
REMARK 4.1 Theorem 4.1 extends without difficulty to more elaborate single-particle operators

$$
T^{(k)}=\frac{1}{2}\left(-\mathrm{i} \nabla_{x^{(k)}}-A(t)\right)^{2}+U\left(x^{(k)}\right)
$$

with a smooth, bounded potential $U$. The extension to the situation (1.3) with a Coulomb potential encounters, however, technical difficulties in estimating the commutators, which we do not address here.

\section{Acknowledgement}

We thank an anonymous referee for a valuable comment that helped to reduce the Sobolev regularity requirement in Theorem 4.1 .

This work was supported by the Austrian Academy of Sciences, APART program, and by DFG, SPP 1324.

\section{REFERENCES}

[1] C. Bardos, I. Catto, N. Mauser, and S. Trabelsi. Analysis of the MCTDHF equations. 2008. Submitted.

[2] M. H. Beck, A. Jäckle, G. A. Worth, and H.-D. Meyer. The multiconfiguration time-dependent Hartree (MCTDH) method: A highly efficient algorithm for propagating wavepackets. Phys. Rep., 324:1-105, 2000.

[3] M. H. Beck and H.-D. Meyer. An efficient and robust integration scheme for the equations of the multiconfiguration time-dependent Hartree (MCTDH) method. Z. Phys. D, 42:113-129, 1997.

[4] I. Burghardt, H.-D. Meyer, and L. S. Cederbaum. Approaches to the approximate treatment of complex molecular systems by the multiconfiguration time-dependent Hartree method. J. Chem. Phys., 111:2927-2939, 1999.

[5] J. Caillat, J. Zanghellini, M. Kitzler, W. Kreuzer, O. Koch, and A. Scrinzi. Correlated multielectron systems in strong laser pulses - an MCTDHF approach. Phys. Rev. A, 71:012712, 2005.

[6] E. Cances and C. Le Bris. On the time-dependent Hartree-Fock equations coupled with a classical nuclear dynamics. Math. Models Methods Appl. Sci., 9:963-990, 1999.

[7] J.M. Chadam and R.T. Glassey. Global existence of solutions to the Cauchy problem for time-dependent Hartree equations. J. Math. Phys., 16:1122-1130, 1975.

[8] P.A.M. Dirac. Note on exchange phenomena in the Thomas atom. Proc. Cambridge Phil. Soc., 26:376-385, 1930.

[9] J. Frenkel. Wave Mechanics, Advanced General Theory. Clarendon Press, Oxford, 1934.

[10] E. Hairer, C. Lubich, and G. Wanner. Geometric Numerical Integration, 2nd ed., Springer-Verlag, Berlin, 2006.

[11] E. Hairer, S.P. Nørsett, and G. Wanner. Solving Ordinary Differential Equations I. Springer-Verlag, Berlin, 1987.

[12] T. Kato. Perturbation Theory for Linear Operators. Springer-Verlag, Berlin-Heidelberg-New York, 1966.

[13] T. Kato and H. Kono. Time-dependent multiconfiguration theory for electronic dynamics of molecules in an intense laser field. Chem. Phys. Lett., 392:533-540, 2004.

[14] M. Kitzler, J. Zanghellini, Ch. Jungreuthmayer, M. Smits, A. Scrinzi, and T. Brabec. Ionization dynamics of extended multielectron systems. Phys. Rev. A, 70:041401(R), 2004.

[15] O. Koch. Numerical solution of the time-dependent Schrödinger equation in ultrafast laser dynamics. WSEAS Trans. Math., 3:584-590, 2004.

[16] O. Koch, W. Kreuzer, and A. Scrinzi. MCTDHF in ultrafast laser dynamics. AURORA TR-2003-29, Inst. for Appl. Math. and Numer. Anal., Vienna Univ. of Technology, Austria, 2003. Available at http: //www . othmar-koch.org/research.html.

[17] O. Koch and C. Lubich. Dynamical low rank approximation. SIAM J. Matrix Anal. Appl., 29:434-454, 2007.

[18] O. Koch and C. Lubich. Regularity of the multi-configuration time-dependent Hartree approximation in quantum molecular dynamics. M2AN Math. Model. Numer. Anal., 41:315-331, 2007.

[19] L.D. Landau and E.M. Lifshitz. Quantum Mechanics: Non-Relativistic Theory. Pergamon Press, Oxford-New York, 3rd edition, 1977. 
[20] C. Lubich. A variational splitting integrator for quantum molecular dynamics. Appl. Numer. Math., 48:355-368, 2004.

[21] C. Lubich. On splitting methods for Schrödinger-Poisson and cubic nonlinear Schrödinger equations. Math. Comp., 77:2141-2153, 2008.

[22] C. Lubich. From Quantum to Classical Molecular Dynamics: Reduced Models and Numerical Analysis. Zurich Lectures in Advanced Mathematics. European Mathematical Society, Zurich, 2008.

[23] H.-D. Meyer, U. Manthe, and L. S. Cederbaum. The multi-configurational time-dependent Hartree approach. Chem. Phys. Lett., 165:73-78, 1990.

[24] H.-D. Meyer and G. A. Worth. Quantum molecular dynamics: Propagating wavepackets and density operators using the multi-configuration time-dependent Hartree (MCTDH) method. Theo. Chem. Acc., 109:251-267, 2003.

[25] M. Nest, T. Klamroth, and P. Saalfrank. The multiconfiguration time-dependent Hartree-Fock method for quantum chemical calculations. J. Chem. Phys., 122:124102, 2005.

[26] A. Pazy. Semigroups of Linear Operators and Applications to Partial Differential Equations. Springer-Verlag, New York, 1983.

[27] M. Reed and B. Simon. Methods of Modern Mathematical Physics II: Fourier Analysis, Self-Adjointness. Academic Press, New York-San Francisco-London, 1975.

[28] I. Segal. Non-linear semi-groups. Ann. of Math., 78:339-364, 1963.

[29] J. Zanghellini, M. Kitzler, T. Brabec, and A. Scrinzi. Testing the multi-configuration time-dependent Hartree-Fock method. J. Phys. B: At. Mol. Phys., 37:763-773, 2004.

[30] J. Zanghellini, M. Kitzler, C. Fabian, T. Brabec, and A. Scrinzi. An MCTDHF approach to multi-electron dynamics in laser fields. Laser Physics, 13(8):1064-1068, 2003. 